\title{
Terapia Biológica em Outras Doenças Auto-Imunes
}

\section{Therapies In Other Systemic Autoimmunes Diseases}

\begin{abstract}
Boris A. Cruz ${ }^{(1)}$
Como especialidade médica, a reumatologia experimenta na última década um grande progresso no que diz respeito ao tratamento de seus pacientes. O desenvolvimento dos ditos agentes biológicos mudou a evolução clínica dos pacientes com artrite reumatóide e espondiloartropatias. Além de eficácia sintomática, a terapia biológica apresenta potencial para modificação significativa do curso dessas doenças, incluindo a possibilidade de remissão. Os resultados surpreendentes em artropatias inflamatórias trazem a perspectiva de que agentes biológicos podem também ser de valia em outras doenças sistêmicas auto-imunes, e a literatura médica já aponta resultados positivos em casos refratários e situações peculiares. O presente artigo tem como objetivo apresentar artigos científicos selecionados que discutem o uso de terapia biológica em outras doenças auto-imunes que não artrite reumatóide e espondiloartropatias. No entanto, é importante salientar que essas são indicações ainda não oficializadas pelas agências reguladoras no Brasil, nos Estados Unidos ou na Europa, pelo que seu uso só se justifica em situações especiais, incluindo o devido esclarecimento e o consentimento, preferencialmente por escrito, do paciente ou de seu responsável legal.
\end{abstract}

Ramos-Casals M, García-Hernández FJ, Martínez-Berriotxoa A. Off-Label Use of Biological Therapies in Systemic Autoimmune Diseases (SAD). Description of 1.140 Registered Cases (International Registry of Biological Agents in SAD, iBIOGEAS). [Uso "off-label" de terapias biológicas em doenças sistêmicas auto-imunes. Descrição de 1.140 casos registrados (registro internacional de agentes biológicos em doenças sistêmicas auto-imunes, iBIOGEAS)]. Arthritis Rheum 56(Suppl):S647, 2007. BIOGEAS study group, Hospital Clinic, Barcelona, Espanha.

Esse trabalho descreve os dados de um registro internacional do uso de agentes biológicos em doenças reumáticas que não artrite reumatóide e espondiloartropatias. Quinhentos e trinta pacientes receberam infliximabe, 328 , rituximabe, 260, etanercept, 13, anakinra, oito, adalimumabe, um, ocrelizumabe. Os diagnósticos incluíram síndrome de Sjögren: (SS) ( $\mathrm{n}=216$ casos), granulomatose de Wegener (GW) $(\mathrm{n}=183)$, sarcoidose $(\mathrm{n}=181)$, lúpus eritematoso sistêmico (LES) $(\mathrm{n}=149)$, doença de Behçet $(\mathrm{n}=133)$, doença de Still $(\mathrm{n}=92)$, crioglobulinemia $(\mathrm{n}=86)$ e outras doenças. Os maiores índices de resposta terapêuica ocorreram com o uso de rituximabe em LES (92\%), SS (90\%), síndrome antifosfolípide (100\%), miopatias inflamatórias
(100\%) e vasculites (100\%), para infliximabe em sarcoidose (99\%), doença de Still (89\%) e policondrite recidivante (86\%) e para etanercept em doença de Behçet (96\%). Excesso de efeitos adversos (> 50\% dos casos relatados) foi observado em pacientes que receberam infliximabe para miopatia inflamatória e sarcoidose e etanercept para miopatias inflamatórias. Os autores têm o cuidado de concluir que os dados são retrospectivos e não controlados, pelo que a eficácia desses medicamentos nessas condições clínicas deve ser confirmada em ensaios clínicos. No entanto, torna-se evidente que já existe experiência clínica que justifique o uso desses agentes em condições especiais como refratariedade ao tratamento usual. 
Mok CC, Ho LY, To CH. Rituximab for refractory polymyositis: an open-label prospective study. (Rituximabe para polimiosite refratária: estudo aberto prospectivo). J Rheumatol 34:1864-8, 2007. Tuen Mun Hospital, Hong Kong, China.

Nesse estudo aberto, os autores avaliaram o efeito de rituximabe em quatro pacientes com polimiosite descrita como refratária. Os pacientes apresentavam doença ativa nos últimos dois anos, caracterizada pela persistência de fraqueza proximal, enzimas musculares elevadas e achados de inflamação muscular na eletromiografia, a despeito do uso de corticóide e ao menos dois outros imunossupressores. O tempo médio de evolução da doença foi de 4,8 anos. Os pacientes mantiveram o tratamento imunossupressor que vinham usando e rituximabe foi adicionado ao esquema terapêutico na dose de $375 \mathrm{mg} / \mathrm{m}^{2}$ em quatro infusões semanais. Na avaliação, após 28 semanas, todos os pacientes apresentaram melhora clínica e laboratorial. Dois pacientes apresentaram normalização da força muscular e dos níveis séricos de enzimas musculares, caracterizando remissão completa da doença. Os pacientes não apresentaram efeitos adversos como infecções ou reações infusionais, e o protocolo não incluía o uso de corticóide quando das infusões. Existem outros relatos de casos e pequenas séries de casos de pacientes com dermatomiosite e polimiosite refratárias que responderam ao uso de rituximabe. Está em curso um ensaio clínico controlado para melhor avaliar o papel de depleção de linfócitos B como alternativa de tratamento em miopatias inflamatórias.

Sfikakis P, Markomichelakis N, Alpsoy E. Anti-TNF therapy in the management of Behçet's disease - review and basis for recommendations. (Terapia anti-TNF no tratamento de doença de Behçet - revisão e bases para recomendações). Rheumatology 46:736-741, 2007. Painel multinacional de especialistas em doença de Behçet.

Doença de Behçet é uma doença sistêmica, auto-imune, classificada como vasculite. Sua apresentação clínica é diversa. A inflamação ocular pode levar à cegueira, e o envolvimento visceral como do trato gastrointestinal ou do sistema nervoso central pode levar não só a importante morbidade, mas também ao óbito. Existem poucos ensaios clínicos que apontem tratamentos comprovadamente eficazes em doença de Behçet. A literatura médica recente apresenta uma série de trabalhos que sugerem ser eficaz a terapia anti-TNF em pacientes com doença refratária aos imunossupressores convencionais. Esse artigo é o resultado de um painel de especialistas que propõem um consenso sobre o papel que a terapia anti-TNF pode ocupar no contexto das diferentes opções terapêuticas para o tratamento da doença de Behçet. O resumo das recomendações é mostrado na tabela 1 :

TABELA 1

RECOMENDAÇÕES PARA A PRESCRIÇÃO DE AGENTES ANTI-TNF EM PACIENTES COM DOENÇA DE BEHÇET

\begin{tabular}{|c|c|}
\hline Condição clínica & Recomendação \\
\hline \multicolumn{2}{|l|}{ Inflamação ocular } \\
\hline - Segmento posterior & $\begin{array}{l}\text { Em pacientes com duas a três recidivas/ano, a despeito do uso de/ou intolerantes à azatioprina, à ciclosporina, } \\
\text { ao interferon associados à prednisona, infliximabe pode ser utilizado. Em casos novos, quando de envolvimento } \\
\text { unilateral com grave perda da acuidade visual }(<0,2) \text { ou envolvimento bilateral, infliximabe pode ser usado como } \\
\text { primeira opção }\end{array}$ \\
\hline - Segmento anterior & Não recomendado \\
\hline $\begin{array}{l}\text { Envolvimento parenquimatoso do sistema } \\
\text { nervoso central }\end{array}$ & $\begin{array}{l}\text { Em pacientes com inflamação refratária à ciclofosfamida e prednisona ou em pacientes que apresentam recidiva a } \\
\text { despeito do tratamento de manutenção com azatioprina, infliximabe pode ser utilizado }\end{array}$ \\
\hline Inflamação intestinal & $\begin{array}{l}\text { Em pacientes que falharam ao menos dois imunossupressores dentre azatioprina, ciclosporina, metotrexato, } \\
\text { colchicina, talidomida e interferon e que requerem doses de prednisona }>7,5 \mathrm{mg} / \mathrm{dia} \text {, infliximabe pode ser tentado }\end{array}$ \\
\hline Envolvimento de vasos de maior calibre & Sem informações suficientes para recomendação \\
\hline Manifestações mucocutâneas & $\begin{array}{l}\text { Em pacientes com comprometimento significativo da qualidade de vida e doença em atividade a despeito do uso de } \\
\text { azatioprina, colchicina ou talidomida e necessitando de doses de prednisona }>7,5 \mathrm{mg} \text {, etanercept ou infliximabe } \\
\text { podem ser utilizados }\end{array}$ \\
\hline Artrite & $\begin{array}{l}\text { Em pacientes que falharam ao menos dois imunossupressores dentre azatioprina, ciclosporina, metotrexato, } \\
\text { colchicina, talidomida e interferon e que requerem doses de prednisona }>7,5 \mathrm{mg} / \mathrm{dia} \text {, infliximabe pode ser tentado }\end{array}$ \\
\hline
\end{tabular}


Gunnarsson I, Sundelin B, Jonsdottir T. Histopathologic and clinical outcome of rituximab treatment in patients with cyclophosphamide-resistant proliferative lupus nephritis. (Desfecho clínico e histopatológico de pacientes com nefrite lúpica proliferativa resistente à ciclofosfamida tratados com rituximabe). Arthritis Rheum 4:1263-72, 2007. Karolinska University Hospital at Solna, Estocolmo, Suécia.

Nesse estudo, os autores trataram consecutivamente sete pacientes do sexo feminino com lúpus e nefrite proliferativa definida como refratária à ciclofosfamida, tanto clínica quanto histologicamente. O esquema terapêutico incluiu a manutenção da ciclofosfamida em duas infusões de $0,5 \mathrm{~g} / \mathrm{m}^{2}$ de superfície corporal com intervalo de 30 dias, corticóide endovenoso seguido de corticóide oral e rituximabe, $375 \mathrm{mg} / \mathrm{m}^{2}$, em quatro infusões semanais. A seguir, os pacientes tinham sua dose de corticóide progressivamente reduzida de acordo com a evolução clínica, mas não faziam uso de nenhum outro agente imunossupressor. Após seis meses, existiu melhora no índice SLEDAI, melhora da função renal, redução significativa nos níveis séricos de anticorpos anti-DNA, melhora dos índices de atividade e da classe histológica em nova biópsia. Três pacientes apresentaram remissão renal completa e uma paciente, remissão parcial. Ainda que não tenham alcançado critérios para remissão, os outros três pacientes apresentaram melhora clínica significativa. No que diz respeito à segurança, um paciente apresentou erupção eritematosa fotossensível, um apresentou herpes zóster limitado, um apresentou infecção do trato urinário não complicada, e um, neutropenia febril, após a segunda infusão de ciclofosfamida. Mesmo em uma amostra pequena, a confirmação de melhora clínica e histológica é um dado relevante. Ainda que estudos com maior casuística e principalmente maior tempo de seguimento sejam necessários para se estabelecerem recomendações quanto ao uso de rituximabe em nefrite lúpica, os autores entendem que esses dados sugerem que essa é uma alternativa válida para pacientes com envolvimento renal refratário aos esquemas terapêuticos convencionais com ciclofosfamida.

Smith KGC, Jones RB, Burns SM, Jayne DRW. Long-term comparison of rituximab treatment for refractory systemic lupus erythematosus and vasculitis: remission, relapse, and re-treatment. (Comparação do tratamento com rituximabe em longo prazo para lúpus eritematoso sistêmico e vasculites refratárias: remissão, recidiva e retratamento). Arthritis Rheum 9:2970-82, 2006. Addenbrooke's Hospital, Cambridge, Reino Unido.

Em um estudo prospectivo, os autores incluíram 11 pacientes com lúpus e 11 pacientes com vasculites associadas ao ANCA (granulomatose de Wegener, $\mathrm{n}=5$; poliangeíte microscópica, $\mathrm{n}=5$; síndrome de Churg-Strauss, $\mathrm{n}=1$ ) com doença ativa e/ou refratária ao tratamento convencional. Esses pacientes receberam rituximabe, 375 $\mathrm{mg} / \mathrm{m}^{2}$, em quatro infusões, além de ciclofosfamida, 500 $\mathrm{mg}$, em infusão única junto à primeira infusão de rituximabe. Existiu resposta clínica e laboratorial em 100\% dos pacientes com lúpus e $91 \%$ dos pacientes com vasculites associadas ao ANCA (um dos pacientes com granulomatose de Wegener apresentou progressão do envolvimento de vias aéreas superiores e inferiores, necessitando ciclofosfamida). Após período de observação de 24 meses, sete dos 11 pacientes com lúpus apresentaram recidiva, em média 12 meses após o tratamento. Dos dez pacientes com vasculite que alcançaram remissão, seis apresentaram recida 16,5 meses em média após o tratamento. Desses 13 pacientes que apresentaram recidiva, 11 receberam um segundo curso de rituximabe, alcançando remissão novamente. Após retratamento, nova recidiva ocorreu em média 7,5 meses depois. Três pacientes receberam três ciclos de rituximabe, e um paciente recebeu quatro ciclos em um total de 15 retratamentos. Desses, apenas um não apresentou remissão completa. De maneira geral, rituximabe foi bem tolerado. Os efeitos adversos mais comuns foram reações infusionais leves. Quatro pacientes apresentaram infecções graves, mas tais infecções são freqüentes em um grupo de pacientes, independente do tratamento com rituximabe. Os autores sugerem que a resposta ao rituximabe nesse estudo aponte para uma alternativa de tratamento necessária e relativamente segura em pacientes que esgotaram as alternativas convencionais de tratamento. 
Suhler EB, Smith JR, Wertheim MS. A Prospective Trial of Infliximab Therapy for Refractory Uveitis: Preliminary Safety and Efficacy Outcomes. (Um ensaio prospectivo de tratamento com infliximabe para uveíte refratária: desfechos de segurança e eficácia preliminares). Arch Ophthalmol 123:903-12, 2005. Departamento de Oftalmologia, Portland Veterans Affairs Medical Center, Portland, Estados Unidos.

Neste estudo prospectivo de fase II, 23 pacientes com uveíte classificada como refratária receberam infliximabe na dose de $3 \mathrm{mg} / \mathrm{kg}$ se estivessem em uso de outros agentes imunossupressores $(\mathrm{n}=20)$ ou $5 \mathrm{mg} / \mathrm{kg}(\mathrm{n}=3)$ como monoterapia nos dias 0,14 e 42 , com avaliação de eficácia dez semanas após o início do tratamento. Os pacientes deveriam apresentar uveíte grave o suficiente para ameaçar a acuidade visual e refratária à prednisona $1 \mathrm{mg} / \mathrm{kg}$ e ao menos um agente imunossupressor. Os diagnósticos eram uveíte idiopática $(\mathrm{n}=9)$, doença de Behçet $(\mathrm{n}=4)$, sarcoidose $(\mathrm{n}=3)$, retinopatia birdshot $(\mathrm{n}=3)$, pars planitis $(\mathrm{n}=$ 2), doença de Crohn $(\mathrm{n}=1)$ e coroidite multifocal $(\mathrm{n}=1)$. Dezoito pacientes (78\%) apresentaram critérios de resposta na décima semana que incluíam melhora de ao menos um e piora de nenhum item do composite de acuidade visual, inflamação ocular ao exame oftalmológico, redução de medicação concomitante e sinais inflamatórios na avaliação pela angiofluoresceinografia e/ou tomografia de coerência ocular. Dos pacientes inicialmente acompanhados, 14 mantiveram o tratamento por um ano. Destes, sete $(50 \%)$ mantiveram a boa resposta, cinco deixaram o tratamento por efeitos adversos e dois deixaram o estudo por razões externas. Curiosamente, a freqüência de eventos adversos foi maior do que a prática clínica observa em outras doenças auto-imunes e incluíram-se eventos tromboembólicos $(\mathrm{n}=3)$, reação lúpus-símile $(\mathrm{n}=2)$, insuficiência cardíaca congestiva $(\mathrm{n}=\mathrm{l})$, neoplasia $(\mathrm{n}=\mathrm{l})$ e hemorragia vítrea $(\mathrm{n}=2)$. Os autores discutem que provavelmente a maior parte dessas complicações não poderia ser explicada pelo tratamento e seriam manifestações de condições clínicas que antecederam o estudo como uma embolia pulmonar em uma paciente obesa e com história pregressa de trombose venosa em membros inferiores ou a verificação de estenose coronariana aterosclerótica em um paciente que apresentou infarto miocárdico. Outros estudos menores não controlados também mostraram resultados positivos com infliximabe em pacientes nas mesmas condições. Existem relatos isolados de efeito benéfico com adalimumabe, mas os dados com etanercepte são conflitantes quanto à eficiência em doença inflamatória ocular.

Baughman RP, Drent M, Kavuru M. Infliximab therapy in patients with chronic sarcoidosis and pulmonar involvement. (Infliximabe em pacientes com sarcoidose crônica e envolvimento pulmonar). Am J Respir Crit Care Med 174:795-802, 2006. University of Cincinnati Medical Center, Cincinnati, Estados Unidos.

Este ensaio clínico incluiu 138 pacientes com sarcoidose e envolvimento pulmonar caracterizado por capacidade vital forçada entre $50 \%$ e $85 \%$ do previsto e escala de dispnéia do Medical Research Council de ao menos 1, a despeito do tratamento com prednisona (dose $\geq 10 \mathrm{mg} / \mathrm{dia}$ ) ou ao menos um agente imunossupressor nos três meses que antecederam a triagem. Os pacientes foram randomizados para receber infliximabe nas doses de $3 \mathrm{mg} / \mathrm{kg}, 5 \mathrm{mg} / \mathrm{kg}$ ou placebo nas semanas $0,2,6,12,18$ e 24 , e foram seguidos até a semana 54 . Os pacientes que receberam infliximabe apresentaram aumento em média de $2,5 \%$ na capacidade vital na semana 24 , enquanto os pacientes que receberam placebo se mantiveram estáveis $(\mathrm{p}=0,038)$. No entanto, não existiu melhora em outros parâmetros avaliados como medidas de dispnéia ou teste da caminhada. Em análise estraficada post hoc, pacientes com doença de maior duração e parâmetros de pior gravidade como menor capacidade vital forçada ou maior índice de dispnéia no início do estudo apresentaram resposta de maior magnitude. A incidência de efeitos adversos não diferiu entre os três grupos do estudo. Os autores entendem que os dados apresentados neste trabalho não deixam claro qual o papel da terapia anti-TNF em pacientes com sarcoidose. O ganho médio da capacidade vital de $2,5 \%$ é equivalente ao que se consegue com corticóide, mas pacientes mais graves, usualmente sem resposta ao corticóide, apresentaram melhora mais significativa. Existem relatos de caso e séries de caso que sugerem que a terapia anti-TNF é de valia em diferentes formas de apresentação clínica da doença como inflamação ocular, cutânea e no sistema nervoso central. Outros estudos são necessários para se estabelecerem recomendações, pelo que o uso de infliximabe em pacientes com sarcoidose deve ser avaliado individualmente, apenas quando outras alternativas mais tradicionais de tratamento já foram tentadas. 